\title{
A clinico-pathological approach to management of atopic dermatitis
}

\author{
Hui Ling Foo, ${ }^{1}{ }_{M R C P(U K)}$, Hong Liang Tey, ${ }^{1}$ FRCP(Edin
}

\begin{abstract}
Recent research in atopic dermatitis (AD) has identified it to be a heterogeneous inflammatory skin disorder of different endotypes (immune polarisation of T-cell subsets and genetic mutations) underlying various phenotypes (age of onset, ethnicity, disease severity, etc.). The corresponding heterogeneity in underlying patho-mechanisms of the disease has resulted in an impetus towards an endotype-driven management of $\mathrm{AD}$. We propose a practical approach that is based on classifying AD patients into intrinsic and extrinsic phenotypes and their corresponding underlying endotypes. This approach aims to provide a practical method that integrates recent understanding of AD pathogenesis for a targeted endotype-driven management of $\mathrm{AD}$.
\end{abstract}

Ann Acad Med Singap 2021;50:171-3

Keywords: Atopic dermatitis, extrinsic eczema, intrinsic eczema

Recent research in atopic dermatitis (AD) has identified it to be a heterogeneous inflammatory skin disorder of different endotypes (immune polarisation of T-cell subsets and genetic mutations) underlying various phenotypes (age of onset, ethnicity, disease severity, etc.). ${ }^{1,2}$ The corresponding heterogeneity in underlying patho-mechanisms of the disease may explain the failure of effective control of $\mathrm{AD}$ through inhibition of one specific inflammatory pathway in a subset of patients in the recent dupilumab trials, whereby a reduction of Investigator's Global Assessment score to $0-1$ was seen in only $36-38 \%$ of participants. ${ }^{3}$

The new data has nevertheless augmented our understanding of $\mathrm{AD}$, resulting in an impetus towards an endotype-driven management of AD. However, success for "personalised and precise" therapy remains largely in vitro or in silico, partly due to the lack of a practical stratification strategy to meaningfully correlate clinical phenotypes to underlying pathological endotypes. ${ }^{4}$

Among the various ways to classify AD, we perceive that $\mathrm{AD}$ can be most applicably and effectively divided into intrinsic and extrinsic types. This method of classification draws on phenotypic clues through clinical assessment $^{5-7}$ (Table 1), of which the most important clinical feature is the primary integrity of the skin barrier. Primary integrity of the skin can be largely recognised through inspection of non-lesional uninvolved skin in
AD patients. Patients with extrinsic eczema possess a primary epidermal barrier defect, and the resultant surface changes of dryness, scaling and/or flaking. However, patients with intrinsic eczema do not possess a primary epidermal barrier defect, thus displaying healthy skin at non-lesional areas. We propose the following management of $\mathrm{AD}$ based on intrinsic and extrinsic phenotypes and their corresponding underlying endotypes.

Extrinsic eczema is characterised by a primary epidermal barrier defect due to mutation of filaggrin and/ or other epidermal components, resulting in increased transepidermal water loss, allergen penetration, and activation of Th-2 cytokines $^{8}$ (Fig. 1). This underlying pathology results in the clinical appearance of dry, flaky skin in patients with extrinsic eczema. Thus, reconstituting the defective skin barrier with diligent application of hypoallergenic moisturisers and use of gentle cleansers to reduce allergen sensitisation through the defective barrier is the primary cornerstone in management. ${ }^{9}$ If the epidermis is breached, allergen penetrates and inflammation ensues, triggering the inflammatory cascade of atopic dermatitis. At this stage, suppression of subsequent inflammation can then be achieved via inhibition of the Th-2 inflammatory cascade. The most effective biologic agent for $\mathrm{AD}$ hitherto is dupilumab, a human monoclonal antibody that inhibits IL-4 and IL 13 signalling by binding to the IL-4a receptor.

\footnotetext{
${ }^{1}$ Dermatology, National Skin Centre, Singapore

Correspondence: Dr Hong Liang Tey, Dermatology, National Skin Centre, 1 Mandalay Road, Singapore 308205.

Email: hltey@nsc.com.sg
} 


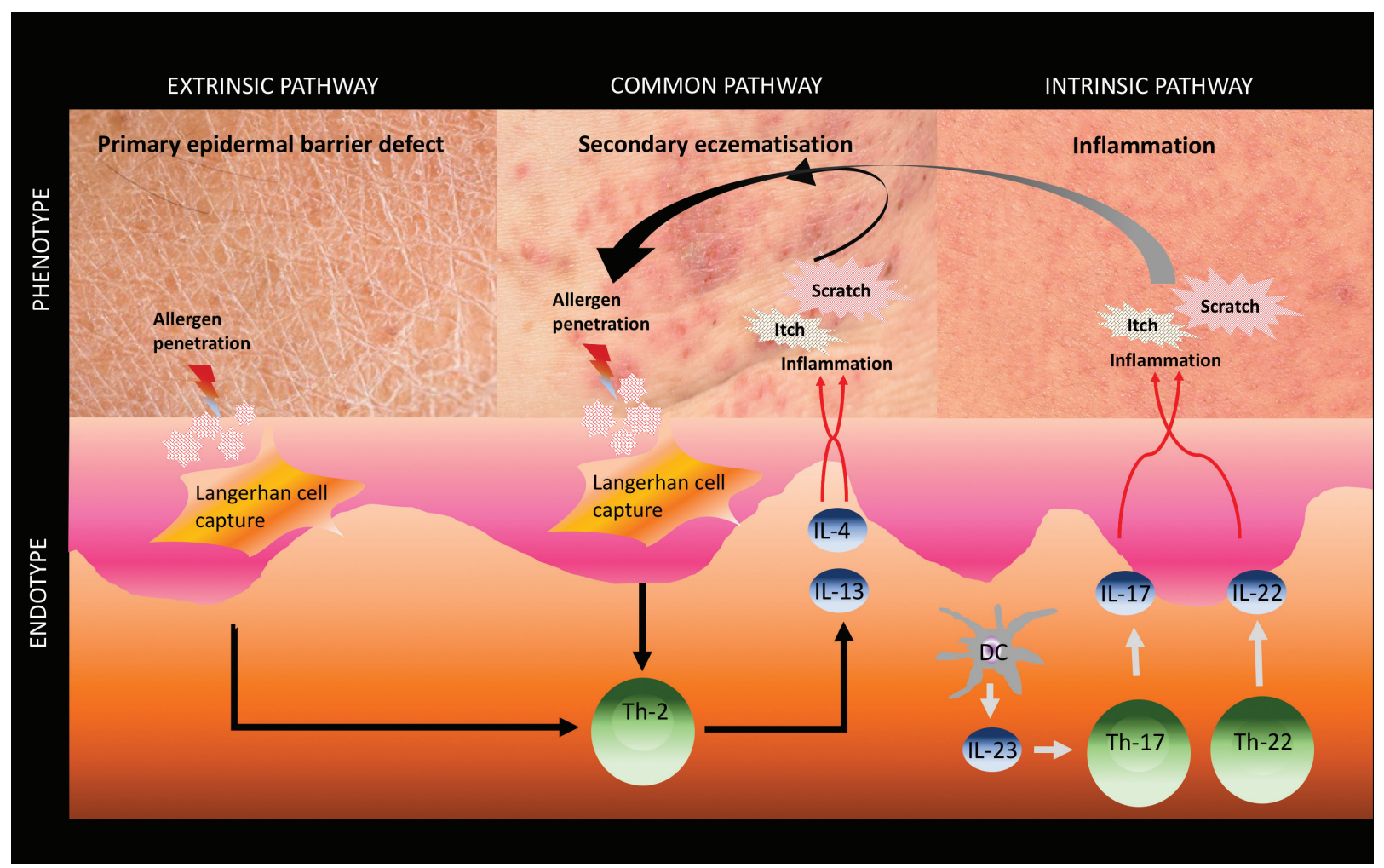

Fig. 1. Putative pathophysiology of extrinsic and intrinsic atopic dermatitis.

Table 1. Clinical features of extrinsic and intrinsic atopic dermatitis (AD)

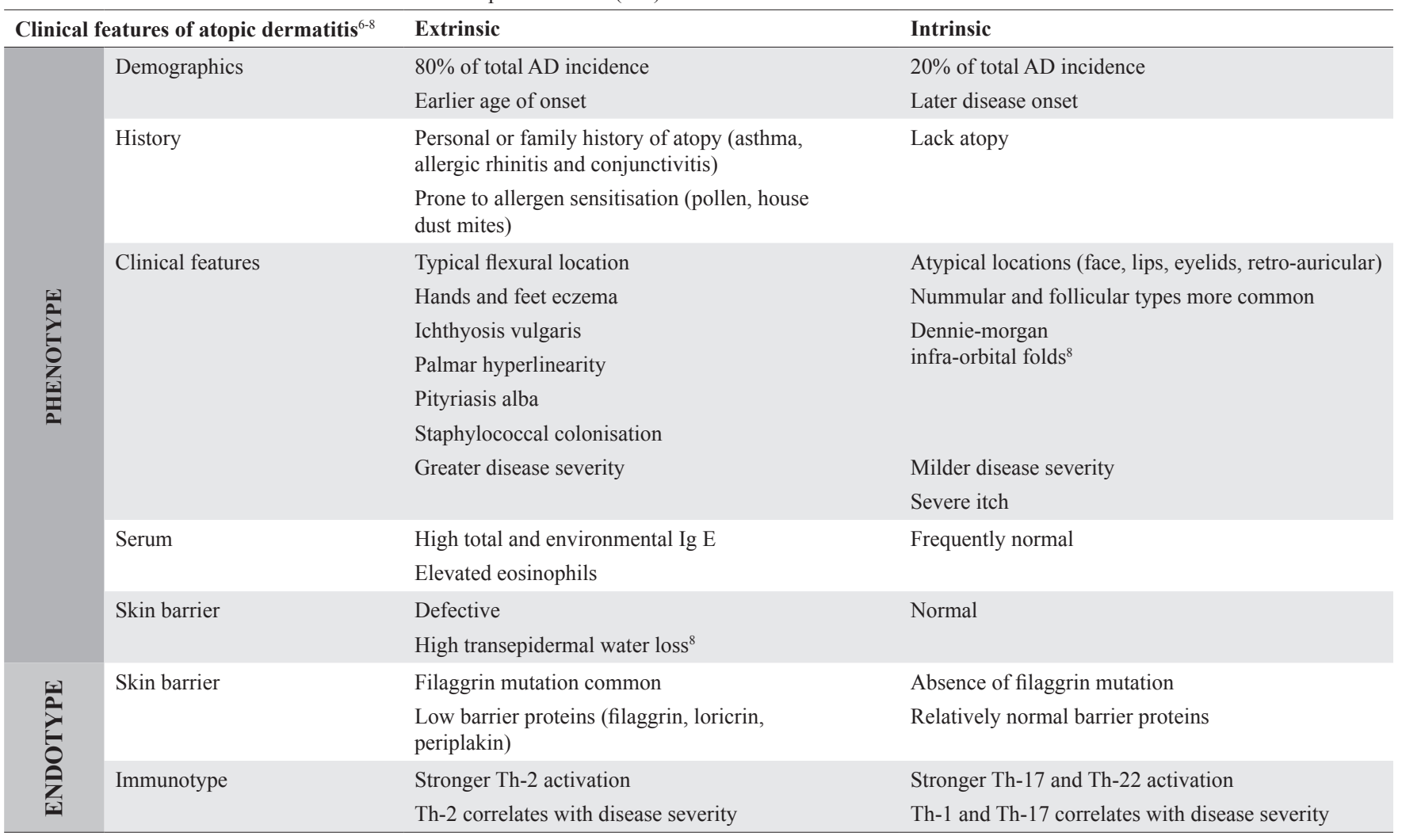

Superscript numbers: Refer to REFERENCES 
The efficacy of sole inhibition of IL-13 (tralokinumab and lebrikizumab) or thymic stromal lymphopoietin (tezepelumab) is still undetermined.

In contrast, patients with intrinsic $\mathrm{AD}$ do not possess a primary epidermal barrier defect. Their primary problem is cutaneous inflammation driven majorly by Th1, Th17 and Th22 T cells. ${ }^{10}$ This causes itch which inevitably leads to scratching, thus creating a secondary barrier defect (Fig.1). Hence, inhibition of the cytokine pathways triggered by the Th1, Th17 and Th22 T cells can potentially be the effective approach to manage intrinsic AD. Biologic trials involving inhibition of IL-17 (secukinumab), IL-23 (ustekinumab) and IL-22 (fezakinumab) are underway. ${ }^{11}$ In a phase II secukinumab trial, a higher percentage of patients with intrinsic eczema achieved EASI-50 score compared to those with extrinsic AD. ${ }^{12,13}$ In addition to anti-inflammatories, we propose simultaneous control of itch being key in the primary management of intrinsic AD. This is to minimise scratchinduced damage to the epidermal barrier and consequent secondary eczematisation, as we postulate that once the secondary barrier defect has occurred, intrinsic AD progresses into an inflammatory "common" pathway similar to that of extrinsic AD (Fig. 1).

The cytokines and inflammatory pathways mentioned have been simplified to enable a practical approach to stratifying patients. Many other cytokines (Th-1, S-100, INF, IL-10) ${ }^{14}$ also play a role in AD pathogenesis. IL-31 has shown a pivotal part in itch, and when inhibited, might potentially contribute to breaking the itch-scratch cycle in $\mathrm{AD}$ patients.

Many authors believe that a pathophysiological- and endotypic-based stratification of patients is the way to move forward in $\mathrm{AD}$ management. ${ }^{15,16}$ However, classifying $\mathrm{AD}$ into extrinsic and intrinsic forms might potentially be challenging in a patient who presents at later stages of the disease (i.e. while in the common pathway), when there are manifestations of overlapping endotypes and phenotypes. While a better understanding of the cytokines involved in $\mathrm{AD}$ pathogenesis has been achieved, there is much work to be done to achieve a targeted, tolerated and effective management of $\mathrm{AD}$. Other gaps in $\mathrm{AD}$ management remain, and these include having head-to-head randomised trials comparing the long-term effectiveness, side effect profile, and cost effectiveness of novel systemic ${ }^{17,18}$ and biologic therapies. Further work in understanding more about geneenvironmental interactions with $\mathrm{AD}$ pathophysiology and treatment is also required. ${ }^{19}$

The concept we have presented is relatively new but we believe it can serve as a practical clinical approach to be built upon as our understanding of $\mathrm{AD}$ pathogenesis and novel biologic agents expands. This fresh perspective will ultimately enable physicians to prescribe personalised and precise treatment for $\mathrm{AD}$, attaining better outcomes with less side effects of conventional non-specific immunosuppressive agents.

\section{REFERENCES}

1. Mu Z, Zhao Y, Liu X, et al. Molecular biology of atopic dermatitis. Clin Rev Allergy Immunol 2014;47:193-218.

2. Czarnowicki T, He H, Krueger JG, et al. Atopic dermatitis endotypes and implications for targeted therapeutics. J Allergy Clin Immunol 2019;143:1-11.

3. Simpson EL, Bieber T, Guttman-Yassky E, et al. Two Phase 3 Trials of Dupilumab versus Placebo in Atopic Dermatitis. N Engl J Med 2016;375:2335-48.

4. Mulick AR, Allen V, Williams HC, et al. Classifying atopic dermatitis: protocol for a systematic review of subtypes (phenotypes) and associated characteristics. J Allergy Clin Immunol 2014;134:818-23.

5. Schmid-Grendelmeier P, Simon D, Simon HU, etal. Epidemiology, clinical features, and immunology of the "intrinsic" (non-IgE-mediated) type of atopic dermatitis (constitutional dermatitis). Allergy 2001;56:841-9.

6. Silverberg NB. Typical and atypical clinical appearance of atopic dermatitis. Clin Dermatol 2017;35:354-9.

7. Pugliarello S, Cozzi A, Gisondi Pet al. Phenotypes of atopic dermatitis. Dtsch Dermatol Ges 2011;9:12-20.

8. Tokura Y. Extrinsic and intrinsic types of atopic dermatitis. Review J Dermatol Sci 2010;58:1-7.

9. Simpson EL, Chalmers JR, Hanifin JM et al. Emollient enhancement of the skin barrier from birth offers effective atopic dermatitis prevention. J Allergy Clin Immunol 2014;134818-23.

10. Suárez-Fariñas M, Dhingra N, Gittler J, et al. Intrinsic atopic dermatitis (AD): shows similar Th2 and higher Th17 immune activation compared to extrinsic AD. J Allergy Clin Immunol 2013;132:361-70.

11. Czarnowicki T, He H, Krueger JG et al. Atopic dermatitis endotypes and implications for targeted therapeutics. J Allergy Clin Immunol 2019;143:1-11.

12. Vakharia PP, Silverberg J. New therapies for atopic dermatitis: Additional treatment classes. J Am Acad Dermatol 201878(3 Suppl 1):S76-S83.

13. Wu J, Guttman-Yassky E. Efficacy of biologics in atopic dermatitis. Expert Opin Biol Ther 2020;20:525-38.

14. Mansouri Y, Guttman-Yassky E. Immune Pathways in Atopic Dermatitis, and Definition of Biomarkers through Broad and Targeted Therapeutics. J Clin Med 2015;4:858-73.

15. Cabanillas B, Brehler AC, Novak N. Atopic dermatitis phenotypes and the need for personalized medicine. Curr Opin Allergy Clin Immunol 2017;17:309-15.

16. Bieber T, D'Erme AM, Akdis CA, et al. Clinical phenotypes and endophenotypes of atopic dermatitis: Where are we, and where should we go? J Allergy Clin Immunol 2017;139(4S):S58-S64.

17. S S Lee, A W H Tan, Y C Giam. Cyclosporin in the treatment of severe atopic dermatitis: a retrospective study. Ann Acad Med Singap 2004;33:311-3.

18. M Bigby. New treatment for atopic dermatitis--facts, comparisons and uncertainties. Ann Acad Med Singap 2005;34:650-1.

19. Alexander H, Patton T, Jabbar-Lopez ZK, et al. Novel systemic therapies in atopic dermatitis: what do we need to fulfil the promise of a treatment revolution? Review F1000Res 2019 Jan 31;8:F1000 Faculty Rev-132. 